\title{
Embarazo y COVID-19. Consideraciones en la práctica de la hematología
}

Carolina Balderas-Delgado , Álvaro Cabrera-García y Héctor A. Baptista-González²,3*

${ }^{1}$ Hematología/Trasplante de células progenitoras hematopoyéticas, Clínica de Cáncer y Embarazo (CREHER), Hospital Regional de Alta Especialidad de Ixtapaluca, Edo. Méx.; ${ }^{2}$ Hematología Perinatal, Instituto Nacional de Perinatología, Ciudad de México; ${ }^{3}$ Medicina Transfusional y Banco de Sangre, Médica Sur, Ciudad de México. México

\section{Resumen}

La enfermedad por coronavirus 2019 (COVID-19) en una población vulnerable, como es el caso de la mujer embarazada, feto y recién nacido, obliga a establecer estrategias efectivas y seguras centradas en la seguridad del binomio madre-hijo. El objetivo del presente reporte es presentar los resultados de la revisión de las fuentes de información secundaria (metaanálisis y revisión sistemática) del estado del arte en el avance del conocimiento de la COVID-19 durante el embarazo. En diferentes reportes se ha insistido en que la mortalidad materna por COVID-19 es baja. Sin embargo, la razón de mortalidad materna (RMM) aumentó de 30.9 a 45.5 defunciones por cada 100,000 nacimientos, es decir, mostró un incremento del 36.32\% respecto a la misma semana del 2019. Pero el tema no se limita a la COVID-19, el aumento en la RMM es del 24.\% para hemorragia materna, del $20 \%$ para la enfermedad hipertensiva y del $28.5 \%$ para sepsis puerperal. Sin embargo, por su naturaleza de condición inédita y el comportamiento particular de la COVID-19 durante el periodo perinatal, la generación de nuevos datos, su integración a información accesible y su análisis clínico epidemiológico inevitablemente proporcionarán nuevas evidencias que deberán integrarse a la gestión y práctica clínica. No existe un comportamiento hematológico característico o de las complicaciones trombóticas o hemorrágicas de la paciente con COVID-19, son características clínicas similares a las que se presenta en sus pares sin embarazo. El aumento global en todas las causas de mortalidad materna no son exclusivas de la COVID-19, lo que expone las deficiencias del sistema de salud en términos de atención primaria de la salud, vigilancia prenatal y planificación familiar, entre otros programas más; adicional al impacto de la COVID-19. Es una necesidad imperiosa el rediseño de las políticas públicas en términos de atención primaria para la salud a toda la población, en particular para las mujeres embarazadas.

PALABRAS CLAVE: Embarazo. COVID-19. Trombosis venosa.

\section{Pregnancy and COVID-19. Considerations in the practice of Hematology}

\section{Abstract}

Coronavirus disease 2019 (COVID-19) in a vulnerable population, such as the pregnant woman, fetus, and newborn, requires an establishment of effective and safe strategies focused on the safety of the mother-child binomial. The objective of this report is to present the results of the review of secondary information sources (meta-analysis and systematic review), of the state of the art in the advancement of knowledge of the disease due to COVID-19 during pregnancy. Different reports have insisted that maternal mortality from COVID-19 is low. However, the maternal mortality ratio (MMR) increased from 30.9 to 45.5 deaths per 100,000 births, that is, it showed an increase of $36.32 \%$ compared to the same week of 2019. Due to its unprecedented condition and the particular behavior of the COVID-19 disease during the perinatal period, the generation of new data, its integration into accessible information and its epidemiological clinical analysis will inevitably provide new evidence that must be

Correspondencia:

*Héctor A. Baptista-González.

E-mail: baptistagh@gmail.com

0016-3813/@ 2021 Academia Nacional de Medicina de México, A.C. Publicado por Permanyer. Este es un artículo open access bajo la licencia CC BY-NC-ND (http://creativecommons.org/licenses/by-nc-nd/4.0/).
Fecha de recepción: 09-09-2020

Fecha de aceptación: 09-02-202 
integrated into clinical management and practice. But the issue is not limited to COVID-19, the increase in MMR is 24\% for maternal obstetric hemorrhage, $20 \%$ for hypertensive disease, and $28.5 \%$ for puerperal sepsis. There is no characteristic hematological behavior and the appearance of thrombotic or hemorrhagic complications in the patient with COVID-19, without clinical characteristics similar to those seen in her non-pregnant peers. The global increase in all causes of maternal mortality are not exclusive to COVID-19, which exposes the deficiencies of the health system in terms of primary health care, prenatal surveillance, family planning, among other programs; additional to the impact of COVID-19. The redesign of public policies in terms of primary health care for the entire population is an urgent need, particularly for pregnant women.

KEY WORDS: Pregnancy. COVID-19. Venous thrombosis.

\section{Introducción}

La pandemia de la enfermedad por coronavirus 2019 (COVID-19), causada por el coronavirus 2 del síndrome respiratorio agudo grave (SARS-CoV-2), ha tenido un impacto significativo en la población mundial, aunque con variaciones locales. Afecta a población vulnerable por factores de riesgo de comorbilidad' en presencia de determinantes sociales de dispari$\mathrm{dad}^{2} \mathrm{e}$ impacto adicional en la morbilidad y mortalidad del personal de la salud que atiende a este tipo de paciente ${ }^{1}$. La infección por SARS-CoV-2 en el periodo grávido puerperal parece asociarse a mayor riesgo de complicaciones, como parto prematuro, del $20 \%$ para los nacidos por vía vaginal y del $80 \%$ para los obtenidos mediante operación cesárea, datos que están influidos por la práctica obstétrica local ${ }^{3}$. En México adquiere una relevancia significativa, al convertirse en la primera causa de mortalidad materna. Sin embargo, no es clara la evidencia que apoye la transmisión vertical de la infección por SARS-CoV-2 en el feto y es escasa la información sobre el impacto de la COVID-19 en el recién nacido ${ }^{3,4}$. Este panorama merece un llamado de atención para conducir la investigación del comportamiento clínico de la COVID19 durante el periodo grávido puerperal.

Por ser una enfermedad inédita, la cantidad de la evidencia científica se acumula rápidamente y el objetivo del presente reporte es presentar el estado del arte de la COVID-19 en la gestante, el feto y el recién nacido, empleando las fuentes de información secundaria (metaanálisis [MA] y revisión sistemática [RS]), bajo la conciencia clara del rápido avance del conocimiento sobre esta enfermedad.

\section{Cambios fisiológicos de importancia hematológica durante el embarazo}

Todos los componentes de la tríada de Virchow: hipercoagulabilidad, estasis venosa y daño endotelial, ocurren durante el embarazo ${ }^{5}$. Existe un incremento en factores procoagulantes (como el factor de von Willebrand, el factor VIII, el factor V y el fibrinógeno) al mismo tiempo que una resistencia adquirida a la proteína C activada (principal anticoagulante endógeno) y reducción en la proteína $\mathrm{S}^{6}$. Estos cambios se acompañan de alteración en la fibrinólisis mediante un incremento en el inhibidor 1 y 2 del activador del plasminógeno, producidos por la placenta 7 . Estos cambios representan la preparación fisiológica en las pacientes embarazadas para el reto hemostásico que generará el parto. La estasis venosa ocurre al final del tercer trimestre del embarazo y alcanza su pico máximo en la semana $36^{8}$. El daño endotelial a los vasos pélvicos puede ocurrir tanto en el parto vaginal como en la cesárea. Casi el $90 \%$ de los casos de trombosis venosa profunda (TVP) en las mujeres embarazadas afectan la pierna izquierda, en comparación con solo el $55 \%$ en las mujeres no embarazadas $^{8}$. Esta diferencia puede ser el reflejo de la compresión de la vena iliaca izquierda por la arteria iliaca derecha y la arteria ovárica, las cuales cruzan la vena sobre el lado izquierdo solamente. La mayoría de los casos de TVP en el embarazo son trombosis ileofemoral más que de las venas tibiales (72 vs. $9 \%$ ) y la TVP ileofemoral es más probable que la TVP de las venas tibiales para conducir a tromboembolia pulmonar $^{8}$.

\section{Cambios hematológicos durante el periodo grávido puerperal complicado con COVID-19}

Las variables de susceptibilidad o protección para la COVID-19 en las mujeres durante el periodo grávido puerperal provienen de la comparación en otras enfermedades infecciosas epidémicas como la influenza, así como del impacto social que tiene la presencia de la enfermedad en esta etapa de la vida.

No hay un comportamiento clínico particular en las gestantes con COVID-19. La mayor parte de la 
Tabla 1. Cambios hematológicos durante el periodo grávido puerperal complicado con enfermedad por coronavirus 2019 (COVID-19)

\begin{tabular}{|c|c|c|c|c|}
\hline \multirow[t]{2}{*}{ Parámetro } & \multirow[t]{2}{*}{ Gestantes con COVID $(\%)^{4,17-19}$} & \multirow[t]{2}{*}{ Pacientes con COVID $\%\left(\right.$ IC 95\%) ${ }^{10}$} & \multicolumn{2}{|c|}{ Pacientes con COVID $\%($ (IC 95\%) } \\
\hline & & & No severa & Severa \\
\hline Linfopenia & 22 a 59.0 & $57.4(44.8-69.5)$ & 80.4 & 96.1 \\
\hline Neutrofilia & & $25.9(18.6-33.9)$ & & \\
\hline Leucopenia & & $20.1(15.5-27.2)$ & 28.1 & 61.1 \\
\hline Trombocitopenia & 13 & $11.4(5.5-18.7)$ & 31.6 & 57.7 \\
\hline Leucocitosis & 38 & $9.8(5.1-15.5)$ & 4.8 & 11.4 \\
\hline Linfocitosis & & $8.2(0.5-12.5)$ & & \\
\hline Neutropenia & & $3.6(0-12.5)$ & & \\
\hline
\end{tabular}

descripción clínica de la COVID-19 proviene de reportes principalmente en población de origen chino $^{9,10}$ y, en menor medida, de países de habla inglesa ${ }^{11,12}$ y europeos, que se ha sintetizado en metaanálisis de estudios observacionales ${ }^{9,10}$, o con gestantes con prueba confirmatoria de esta enfermedad ${ }^{13}$.

La mayoría de los factores de riesgo descritos en su asociación a mayor mortalidad por COVID-10 (edad avanzada, cáncer, etc.) no aplican para la mujer gestante $y$, fuera de la obesidad ${ }^{14,15}$, no hay suficiente evidencia que sea aplicable de manera generalizada. Hay cierta evidencia que señala que las embarazadas que viven en áreas de mayor densidad poblacional presentan mayor riesgo de adquirir la infección. Esto pudiera ser un elemento de apoyo a las políticas de tamizaje universal de COVID-19 en toda embarazada y así poder identificar los grupos de mayor riesgo de exposición y adquisición de la enfermedad ${ }^{16}$. Sin embargo, en la práctica más del $60 \%$ de las gestantes en quienes se realizó la detección del SARS-CoV-2 al momento de su ingreso hospitalario para la atención del parto o de las complicaciones del embarazo no tenían síntomas de la infección ${ }^{14}$.

A los signos y síntomas habituales, como fiebre, tos, fatiga o mialgias (que se presentan en más de la mitad de los pacientes), se agregan otros que pueden ser la representación hematológica de la enfermedad $^{12}$. En gestantes infectadas por el SARS-CoV-2, la fatiga fue el síntoma más prevalente $(54.5 \%)$, seguido de tos $(50.1 \%)$ y fiebre $(27.6 \%)$. Otros síntomas comunes son disnea, mialgia y dolor de garganta, en aproximadamente el 21,16 y $11 \%$ de las mujeres embarazadas, respectivamente; la prevalencia de diarrea fue inferior al $10 \%{ }^{13}$. La prevalencia de comorbilidades como la hipertensión arterial (incluida hipertensión inducida por el embarazo) y la diabetes (incluida la diabetes gestacional) fue del 3.7 y $4.2 \%$, respectivamente, mientras que el $4.7 \%$ de las mujeres embarazadas con COVID-19 tenían asma ${ }^{13}$.

Los cambios hematológicos en pacientes con COVID-19 son amplios (Tabla 1). Se ha señalado la presencia de leucopenia en el $33.7 \%$, linfopenia en el $83.2 \%$ y trombocitopenia en el $36.2 \%$ de los casos, intentando asociar la linfopenia con el desarrollo de síndrome de dificultad respiratoria aguda o la necesidad de ingreso a la unidad de terapia intensiva (UTI), y en casos confirmados hasta el 48,43 y $36 \%$ de las mujeres embarazadas infectadas tenían niveles elevados de proteína $C$ reactiva $(P C R)$, linfopenia y leucocitosis, respectivamente ${ }^{13}$.

Se han evaluado distintos biomarcadores que puedan tener algún impacto en el pronóstico de la enfermedad, dentro de ellos se han incluido a la proteína $C$ reactiva, amiloide A sérico, interleucina 6 , lactato deshidrogenasa, relación neutrófilos/linfocitos, dímero D (DD), troponina cardiaca, biomarcadores renales, linfocitos y recuento de plaquetas. Todos ellos han mostrado niveles significativamente más altos en pacientes con complicaciones graves por la COVID-19 en comparación con sus contrapartes no graves. Solamente los recuentos de linfocitos y plaquetas presentan valores significativamente más bajos en pacientes graves en comparación con pacientes no graves (Tabla 2).

El análisis de los DD en función de la gravedad de la enfermedad muestra su aumento consistente en pacientes con enfermedad grave ${ }^{20}$. Pero se debe considerar que en la embarazada existe un aumento 
Tabla 2. Cambios bioquímicos durante el periodo grávido puerperal complicado con enfermedad por coronavirus 2019 (COVID-19)

\begin{tabular}{|c|c|c|c|c|}
\hline \multirow[t]{2}{*}{ Parámetro } & \multirow[t]{2}{*}{ Gestantes con COVID (\%) } & \multirow[t]{2}{*}{ Pacientes con COVID $\%\left(\right.$ IC 95\%) ${ }^{10}$} & \multicolumn{2}{|c|}{ Pacientes con COVID $\%($ IC 95\%) } \\
\hline & & & Moderada & Grave \\
\hline Bilirrubinas* & & $14.3(3.1-30.5)$ & 9.9 & 13.3 \\
\hline ALT alta & 11.9 & $14.2(4.6-27.1)$ & 19.8 & 28.1 \\
\hline AST alta & 11.9 & $18.6(8.5-31.1)$ & 18.2 & 39.4 \\
\hline Creatinina alta & & $3.1(0-18.0)$ & & \\
\hline DHL elevada & & $51.6(31.4-71.6)$ & 37.2 & 58.1 \\
\hline PCR aumentada & 45.7 a 70.0 & $68.6(58.2-78.2)$ & 56.4 & 81.5 \\
\hline Dímeros $\mathrm{D}$ altos & & $29.3(4.8-61.6)$ & 43.2 & 59.6 \\
\hline
\end{tabular}

*Hiperbilirrubinemia directa.

IC 95\%: intervalo de confianza del 95\%; ALT: alanina aminotransferasa; AST: aspartato aminotransferasa; DHL: lactato deshidrogenasa; PCR: proteína C reactiva.

paralelo a la edad gestacional en la concentración de los DD, hasta valores promedio superiores a los $1,500 \mathrm{ug} / \mathrm{l}^{21}$. No hay suficiente evidencia que evalúe el impacto de los valores de los DD en embarazadas con COVID-19. En un reporte de serie de casos se halló incremento en la cantidad de DD de 12 a 17 veces los valores normales en embarazadas. Los DD elevados son considerados un marcador de mal pronóstico en pacientes no embarazadas. También se ha reportado ${ }^{22}$ mayor cantidad de DD en aquellos pacientes que requirieron admisión a $\mathrm{UCl}$ vs. aquellos que no $(2.4 \mu \mathrm{g} / \mathrm{mL}$ [0.6-14.4] vs. $0.5 \mu \mathrm{g} / \mathrm{mL}$ [0.3$0.8])$. De manera similar, a partir de estudios observacionales se han reportado valores más elevados de DD en no sobrevivientes vs. sobrevivientes $(2.12 \mu \mathrm{g} / \mathrm{mL}[0.8-5.3 \mu \mathrm{g} / \mathrm{mL}]$ vs. $0.6 \mu \mathrm{g} / \mathrm{mL}$ [0.4-1.3 $\mu \mathrm{g} /$ $\mathrm{mL}$ ). Sin embargo, debido al típico incremento de DD durante la gestación ${ }^{23}$ continúa sin estar claro el valor límite como indicador de mal pronóstico en el embarazo. La International Society on Thrombosis and Haemostasis (ISTH) sugiere que aquellas pacientes con incremento significativo de DD (arbitrariamente aceptado que sea tres a cuatro veces superior del límite normal alto) deben hospitalizarse incluso a pesar de no presentar mayor sintomatología ${ }^{24}$. Aunque la investigación todavía está en sus primeras etapas, el descubrimiento de cómo se comportan los diferentes biomarcadores durante el curso de la enfermedad podría ayudar a identificar la enfermedad grave más temprano y posteriormente, mejorar el pronóstico ${ }^{25}$; la misma ISTH no establece la certidumbre analítica y la utilidad clínica en la determinación de los $\mathrm{DD}$, en la gestante en particular y en general para todos los pacientes con COVID-19 ${ }^{26}$.

\section{Intervenciones en las gestantes con COVID-19}

No existen ensayos clínicos en pacientes embarazadas por razones de seguridad y esto significa que los medicamentos que pueden ser efectivos en la población general no se pueden usar para mujeres embarazadas debido a la falta de conocimiento de los efectos secundarios en esta categoría de personas. La elección de usar un medicamento específico para COVID-19 en el embarazo debe tener en cuenta los beneficios y los posibles eventos adversos en cada caso. En la situación actual de incertidumbre y poco conocimiento sobre el manejo de COVID-19 durante el embarazo, esta descripción general puede proporcionar información útil para los médicos con implicaciones prácticas ${ }^{27}$.

Las diferentes propuestas o guías de intervención para la establecer el estrato de riesgo, referencia y tratamiento de la embarazada con COVID-19 proviene de las conclusiones basadas en el mejor de los casos de las infecciones virales como la influenza. Esto significa que el nivel de evidencia es en consensos institucionales o reportes de series de casos. El Colegio Americano de Obstetras y Ginecólogos ha presentado un algoritmo actualizado diseñado para ayudar a los profesionales de la salud a evaluar y tratar rápidamente a las mujeres embarazadas con exposición conocida o con síntomas congruentes con COVID-19 (https://www.acog.org/-/media/project/acog/acogorg/ files/pdfs/clinical-guidance/practice-advisory/ covid-19-algorithm-spanish-4302020.pdf) ${ }^{28}$.

Existen opiniones encontradas a favor y en contra de iniciar o continuar con la administración de la 
aspirina para prevención o tratamiento en la gestante con COVID-1929,30; tampoco hay consenso en cuanto a la dosis, frecuencia y tiempo de administración. En aquellas embarazadas donde se tiene indicada la administración de aspirina, deberá ser independiente de considerar la COVID-19, debiendo seguir los esquemas preventivos aprobados ${ }^{30}$.

\section{Plasma convaleciente, gammaglobulina}

La evidencia del uso de plasma convaleciente o gammaglobulina polivalente endovenosa en gestantes hospitalizadas por la COVID-19 proviene de casos aislados reportados ${ }^{31,32}$. Los estudios informaron resultados inconsistentes, lo que dificulta la comparación de la seguridad y eficacia de esas intervenciones. No se ha completado ningún estudio clínico controlado que evalúe los beneficios y los daños del plasma convaleciente o la gammaglobulina ${ }^{33}$. Más aún, no hay estudios clínicos registrados sobre el uso de plasma convaleciente en embarazadas o puérperas con COVID-19 en ninguna etapa clínica. De tal suerte que no son intervenciones recomendadas y el clínico deberá considerar cuidadosamente en cada caso en particular el balance de riesgos y beneficios.

\section{Trombosis, embarazo y COVID-19}

Los resultados maternos relacionados con la atención de la COVID-19 han reportado el ingreso a la UTI del $9 \%$ aproximadamente, con una mortalidad relativamente baja (alrededor del 1.2\%). Así mismo, hasta el $25 \%$ de los neonatos han sido atendidos en UTI, en su mayoría por nacimientos pretérmino; en menos del $2 \%$ se ha detectado ARN viral antes de las 12 horas de vida, con reportes extraordinarios de transmisión vertical ${ }^{15}$.

Aunado a esto, la preocupación por las complicaciones relacionadas con el embarazo (que son un problema de salud mundial) ha requerido intervenciones específicas, sobre todo en el contexto de hipertensión durante el embarazo, hemorragia o trombosis perinatal. Esta última es la causa más frecuente de morbimortalidad en países desarrollados, los cuales han diseñado estrategias para limitar los eventos trombóticos en la gestación y el puerperio. Cinco de las más importantes asociaciones expertas en atención obstétrica han recomendado que todas las mujeres en estado grávido deben tener una evaluación de riesgo en cada una de las visitas médicas, con especial atención en el primer trimestre, en la semana 28 de gestación y en el puerperio inmediato ${ }^{34}$. Cada una de estas organizaciones tiene herramientas para determinar si estas pacientes deben tener tromboprofilaxis. Una de las más utilizadas es la realizada por el Colegio Británico de Ginecología y Obstetricia (RCOG), quienes clasifican en riesgo bajo, intermedio $y$ alto tanto en momento prenatal como posnatal ${ }^{35}$.

Todas estas escalas toman en cuenta factores de riesgo trombogénico preexistentes (antecedentes personales, trombofilias conocidas), transitorios y obstétricos, que identifican a mujeres que deben recibir heparina de bajo peso molecular (HBPM) durante el tiempo que permanezcan con riesgo aumentado de trombosis (principalmente los primeros 10 días de puerperio). Algunos de los efectos indeseables de la anticoagulación profiláctica reportados son: alto costo, reacciones en la piel (20-40\% reacciones de hipersensibilidad tipo IV, sangrado), hematomas epidurales y algunos otros, como trombocitopenia inducida por heparina $(<0.1 \%)$ u osteoporosis ${ }^{36}$.

La coagulopatía predominantemente trombogénica existente en las infecciones complicadas por SARS-CoV-2 pone en discusión las decisiones de profilaxis en mujeres gestantes que ya per se tienen un importante estado procoagulante. Recientemente, la Sociedad Española de Trombosis y Hemostasia enfatizó que todas las mujeres embarazadas o en puerperio con COVID-19 confirmada deben recibir tromboprofilaxis independientemente de la gravedad de la sintomatología ${ }^{37}$. Aquellas con sospecha de COVID-19 deberán de ser evaluadas mediante las herramientas habituales de clasificación de riesgo, tomando en cuenta que esta infección viral forma parte de los factores transitorios (infección sistémica actual). El tiempo en el que deben permanecer bajo anticoagulación depende del riesgo asignado (desde el primer trimestre, a partir de la semana 28 de gestación, solo los primeros 10 días de puerperio o hasta el término de este). Algunas otras recomendaciones europeas en este rubro son:

- Si la paciente se encuentra en aislamiento domiciliario, debe garantizarse la tromboprofilaxis durante 14 días, después de este tiempo se reevaluará con las escalas de riesgo para determinar su continuación.

- Todas las mujeres embarazadas hospitalizadas con sospecha de infección por SARS-CoV-2 o confirmadas deben recibir tromboprofilaxis con HBPM, a menos que el parto sea en las próximas 12 horas.

- La dosis correcta de HBPM aún es desconocida, pero se prefieren las dosis estándar sobre las dosis intermedias o bajas. 
Tabla 3. Evaluación hemostásica e intervenciones en la gestante con enfermedad por coronavirus 2019 (COVID-19)

Recomendaciones para la evaluación de la hemostasia en la gestante con COVID-19

- En cada mujer embarazada con COVID-19, confirmada o sospechada, al momento de la evaluación inicial se debe realizar evaluación
sistemática de la hemostasia: conteo de plaquetas, TTPa, tiempo de protrombina, fibrinógeno y dímeros D
Si se encuentra un resultado anormal, efectuar nueva evaluación ante señales de deterioro clínico y si es posible a su ingreso a la sala
de labor
- Recomendaciones para bloqueo neuroaxial
- Plaquetas $>75 \times 10^{3}$, es posible el bloqueo neuroaxial
- Plaquetas > $50 \times 10^{3}$, es posible la anestesia espinal
- TTPa prolongado: detectar anticoagulantes circulantes
- Tiempo de protrombina < $60 \%$ : pruebas complementarias de factores dependientes de la vitamina K

\begin{tabular}{|c|c|c|c|}
\hline \multicolumn{4}{|c|}{ Evaluación de riesgo tromboembólico } \\
\hline \multicolumn{2}{|c|}{$\begin{array}{l}\text { Factores de riesgo de enfermedad tromboembólica en la } \\
\text { gestante con COVID-19 }\end{array}$} & \multicolumn{2}{|r|}{ Profilaxis anteparto } \\
\hline & & Bajo riesgo & No profilaxis \\
\hline $\begin{array}{l}\text { Factores de } \\
\text { riesgo mayor }\end{array}$ & $\begin{array}{l}\text { - Historia personal de ETE trombofilia de } \\
\text { alto riesgo asintomática } \\
\text { - } \mathrm{O}_{2} 4 \text { l/min o HFNO o ventilación mecánica }\end{array}$ & $\begin{array}{l}\text { Riesgo } \\
\text { moderado }\end{array}$ & $\begin{array}{l}\text { Profilaxis con heparina estándar o HBPM (enoxaparina } \\
4,000 \mathrm{c} / 24 \mathrm{~h} \text { ) }\end{array}$ \\
\hline $\begin{array}{l}\text { Factores de bajo } \\
\text { riesgo }\end{array}$ & $\begin{array}{l}\text { IMC }>30, \text { peso }>120 \mathrm{~kg} \\
\text { Inmovilización completa y prolongada Otros }\end{array}$ & Alto riesgo & $\begin{array}{l}\text { HBPM dosis intermedias (enoxaparina } 40 \mathrm{mg} \mathrm{SC} \mathrm{c/12} \mathrm{ho} \\
60 \mathrm{mg} \text { SC UI con peso }>120 \mathrm{~kg}\end{array}$ \\
\hline Bajo riesgo & Ningún factor de riesgo & & Observaciones: \\
\hline $\begin{array}{l}\text { Riesgo } \\
\text { moderado }\end{array}$ & 102 factores de bajo riesgo combinados & & $\begin{array}{l}\text { Duración: hasta recuperación de la enfermedad } \\
\text { No iniciar profilaxis si el nacimiento es inminente. } \\
\text { Considerar la dosis de HBPM en el manejo del nacimiento }\end{array}$ \\
\hline Alto riesgo & Al menos un factor mayor $y \geq 3$ factores de $n$ & enor riesgo & y del bloqueo neur \\
\hline
\end{tabular}

ETE: enfermedad tromboembólica; HBPM: heparina de bajo peso molecular; HFNO: oxígeno nasal de alto flujo; TTPa: tiempo de tromboplastina parcial activada; IMC: índice de masa corporal.

Adaptada de Benhamou, et al., $2020^{38}$.

- Al egreso de una paciente embarazada o puérpera con COVID-19 confirmada, esta debe permanecer con anticoagulación al menos por 10 días más y si los factores de riesgo persisten, habrá que continuar con la HBPM por 6 semanas más.

Existen propuestas o guías de diversas asociaciones para la evaluación hemostásica de la gestante con COVID, una de ellas es la propuesta francesa ${ }^{38}[38]$, que considera los factores de riesgo de trombofilia congénita y adquirida, así como la formulación e intensidad de dosis de anticoagulación (Tabla 3).

\section{Mortalidad materna}

La gestante presenta mayor riesgo de adquirir infecciones respiratorias de origen viral y desarrollar neumonía severa, debido a los cambios fisiológicos en el sistema inmunitario y la adaptación cardiovascular propia de esta etapa. La presencia de neumonía varía ampliamente, desde el 0 al $14 \%{ }^{4}$, hasta el extremo del $80.3 \%$ (intervalo de confianza: 68.2-89.4) de las embarazadas ${ }^{39}$. El desarrollo de coagulopatía en embarazadas con COVID-19 está prácticamente ausente en los casos no severos, hasta el $0.6 \%$ en los casos clasificados como severos ${ }^{9}$.

La mortalidad materna por COVID-19 es baja, según reporte de estudios de cohorte $^{14}$. Sin embargo, por su naturaleza de condición inédita y el comportamiento particular de la enfermedad por COVID-19 durante el periodo perinatal, la generación de nuevos datos, su integración a información accesible y su análisis clínico epidemiológico inevitablemente proporcionará nuevas evidencias que deberán integrarse a la gestión y práctica clínica.

De acuerdo con el reporte de la Dirección general de Epidemiología a la semana 50 de registro ${ }^{40}$, la razón de mortalidad materna (RMM) calculada es de 45.5 defunciones por cada 100,000 nacimientos estimados, lo que representa un incremento del $36.32 \%$ respecto a la misma semana del 2019. Las principales causas de defunción confirmadas fueron: COVID-19 (21.0\%), 
Gaceta Médica de México. 2021;157(Supl 3)

Tabla 4. Principales causas de muerte maternas y razón de muerte materna 2019 a 2020 (semana 50)

\begin{tabular}{|c|c|c|c|c|c|c|c|}
\hline \multirow[t]{2}{*}{ Condición } & \multicolumn{3}{|c|}{2019} & \multicolumn{3}{|c|}{2020} & \multirow[t]{2}{*}{ Diferencia en EMM (\%) } \\
\hline & Casos 660 & RMM (30.9) & Lugar & Casos (876) & RMM (45.5) & Lugar & \\
\hline COVID-19 & - & - & - & 184 & 9.6 & 1 & - \\
\hline Hemorragia obstétrica & 133 & 6.2 & 1 & 158 & 8.2 & 2 & 24.4 \\
\hline Enfermedad hipertensiva & 128 & 6.0 & 2 & 144 & 7.5 & 3 & 20.0 \\
\hline Aborto & 58 & 2.7 & 3 & 60 & 3.1 & 4 & 13.0 \\
\hline Probable COVID-19 & - & - & - & 49 & 2.5 & 5 & \\
\hline Enf. respiratorias complicadas & 35 & 1.6 & 4 & 36 & 1.9 & 6 & 15.8 \\
\hline Complicaciones del embarazo & 37 & 1.7 & 5 & 33 & 1.7 & 7 & 0 \\
\hline Sepsis puerperal & 21 & 1.0 & 7 & 26 & 1.4 & 8 & 28.5 \\
\hline Trauma obstétrico & 5 & 0.2 & 8 & 9 & 0.5 & 9 & 60.0 \\
\hline Embolia obstétrica & 23 & 1.1 & 6 & 7 & 0.4 & 10 & -63.0 \\
\hline
\end{tabular}

COVID-19: enfermedad por coronavirus 2019; RMM: razón de mortalidad materna, casos por 100000 nacimientos; EMM: exceso de mortalidad materna.

Adaptada de México, Secretaría de Salud, Dirección General de Epidemiología, $2020^{40}$.

hemorragia obstétrica (18.0\%), enfermedad hipertensiva asociada al embarazo (16.4\%), $6.8 \%$ gestantes con probable COVID-19 (5.3\%) y, finalmente, enfermedades respiratorias $(4.1 \%)$. Sin embargo, como hacia la semana 50 no existe el impacto de la influenza en la mortalidad materna, por lo que se puede asumir que los casos de probable COVID-19 y las enfermedades respiratorias junto con los casos comprobados representarían 266 casos, es decir el $30.6 \%$ de todas las causas. Pero la cuestión no se limita a la COVID-19, el aumento en la RMM es del $24 . \%$ para hemorragia materna, el $20 \%$ para la enfermedad hipertensiva, (18.8\%), de enfermedad hipertensiva (12.5\%) y el $28.5 \%$ para sepsis puerperal, como un reflejo de la mayor carga de mortalidad del exceso de mortalidad materna (Tabla 4).

\section{Conclusiones}

Se pueden identificar diferencias significativas entre el comportamiento de la COVID-19 en mujeres embarazadas y no embarazadas:

- Diferencia en los síntomas comunes de COVID-19; los síntomas de fiebre, tos y disnea, en el 83,82 y $31 \%$, respectivamente frente al 28 , 51 y $21 \%$ de los mismos síntomas en gestantes con infección comprobada.

- La diferencia entre la severidad de la enfermedad y el impacto en mortalidad materna, si bien los estudios de metaanálisis (principalmente en gestantes de origen chino con infección comprobada) indican síntomas relativamente leves. Esta diferencia con síntomas más leves en las pacientes embarazadas con COVID-19 puede explicarse por la edad promedio más joven en comparación a la población general de pacientes con COVID-19.

- La COVID-19 es la principal causa de mortalidad materna en México. Sin embargo, también aumentó más de 15 puntos la RMM global. Este aumento global no es exclusivamente debido a la COVID-19, lo que expone las deficiencias del sistema de salud en términos de atención primaria de la salud, vigilancia prenatal y planificación familiar, entre otros programas, más el impacto adicional de la COVID-19.

- Es una necesidad imperiosa el rediseño de las políticas públicas en términos de atención primaria para la salud a toda la población, en particular para las mujeres embarazadas, así como los criterios para establecer hospitales generales 0 de atención obstétrica, que sean considerados áreas libres de COVID-19.

\section{Conflicto de intereses}

Los autores manifiestan no tener conflicto de intereses alguno.

\section{Financiamiento}

La presente investigación no ha recibido ninguna beca específica de agencias de los sectores público, comercial o con ánimo de lucro. 
Responsabilidades éticas

Protección de personas y animales. Los autores declaran que para esta investigación no se han realizado experimentos en seres humanos ni en animales.

Confidencialidad de los datos. Los autores declaran que han seguido los protocolos de su centro de trabajo sobre la publicación de datos de pacientes.

Derecho a la privacidad y consentimiento infor-

mado. Los autores declaran que en este artículo no aparecen datos de pacientes.

\section{Bibliografía}

1. Bandyopadhyay S, Baticulon RE, Kadhum M, Alser M, Ojuka DK, Badereddin $\mathrm{Y}$, et al. Infection and mortality of healthcare workers worldwide from COVID-19: a systematic review. BMJ Glob Health. 2020;5(12):e003097.

2. Duggan CP, Kurpad A, Stanford FC, Sunguya B, Wells JC. Race, ethnicity, and racism in the nutrition literature: an update for 2020. Am J Clin Nutr. 2020;112(6):1409-14

3. Huntley BJF, Huntley ES, Di Mascio D, Chen T, Berghella V, Chauhan SP. Rates of maternal and perinatal mortality and vertical transmission in pregnancies complicated by severe acute respiratory syndrome coronavirus 2 (SARS-Co-V-2) infection: A systematic review. Obstet Gynecol. 2020;136(2):303-12.

4. Juan J, Gil MM, Rong Z, Zhang Y, Yang H, Poon LC. Effects of coronavirus disease 2019 (COVID-19) on maternal, perinatal and neonatal outcomes: A systematic review. Ultrasound Obstet Gynecol. 2020;56(1):15-27.

5. Ahmed S, Zimba O, Gasparyan AY. Thrombosis in coronavirus disease 2019 (COVID-19) through the prism of Virchow's triad. Clin Rheumatol. 2020;39(9):2529-43

6. Clark P, Brennand J, Conkie JA, McCall F, Greer IA, Walker ID. Activated protein $C$ sensitivity, protein $C$, protein $S$ and coagulation in normal pregnancy. Thromb Haemost. 1998;79(6):1166-70.

7. Siennicka A, Kłysz M, Chełstowski K, Tabaczniuk A, Marcinowska Z, Tarnowska $\mathrm{P}$, et al. Reference values of $\mathrm{D}$-dimers and fibrinogen in the course of physiological pregnancy: The potential impact of selected risk factors-A pilot study. Biomed Res Int. 2020;2020:3192350.

8. Devis $P$, Knuttinen MG. Deep venous thrombosis in pregnancy: inciden$\mathrm{ce}$, pathogenesis and endovascular management. Cardiovasc Diagn Ther. 2017;7(Suppl 3):S309-S319.

9. Guan WJ, Ni ZY, Hu Y, Liang WH, Ou CQ, He JX, et al. Clinical characteristics of coronavirus disease 2019 in China. N Engl J Med. 2020;382(18):1708-20.

10. Fu L, Wang B, Yuan T, Chen X, Ao Y, Fitzpatrick T, et al. Clinical characteristics of coronavirus disease 2019 (COVID-19) in China: A systematic review and meta-analysis. J Infect. 2020;80(6):656-65.

11. Pettirosso E, Giles M, Cole S, Rees M. COVID-19 and pregnancy: A review of clinical characteristics, obstetric outcomes and vertical transmission. Aust N Z J Obstet Gynaecol. 2020;60(5):640-59.

12. Akhtar H, Patel C, Abuelgasim E, Harky A. COVID-19 (SARS-CoV-2) infection in pregnancy: A systematic review. Gynecol Obstet Invest. 2020:85(4):295-306.

13. Yee J, Kim W, Han JM, Yoon HY, Lee N, Lee KE, et al. Clinical manifestations and perinatal outcomes of pregnant women with COVID-19: a systematic review and meta-analysis. Sci Rep. 2020;10(1):18126.

14. Khoury R, Bernstein PS, Debolt C, Stone J, Sutton DM, Simpson LL, et al. Characteristics and outcomes of 241 births to women with severe acute respiratory syndrome coronavirus 2 (SARS-CoV-2) infection at five New York City Medical Centers. Obstet Gynecol. 2020;136(2):273-82.

15. Knight M, Bunch K, Vousden N, Morris E, Simpson N, Gale C, et al. Characteristics and outcomes of pregnant women admitted to hospital with confirmed SARS-CoV-2 infection in UK: national population based cohort study. MJ. 2020;369:m2107

16. Emeruwa UN, Ona S, Shaman JL, Turitz A, Wright JD, Gyamfi-Bannerman $C$, et al Associations between built environment, neighborhood socioeconomic status, and SARS-CoV-2 infection among pregnant women in New York City. JAMA. 2020;324(4):390-2

17. Dashraath $P$, Wong JLJ, Lim MXK, Lim LM, Li S, Biswas A, et al. Coronavirus disease 2019 (COVID-19) pandemic and pregnancy. Am J Obstet Gynecol. 2020;222(6):521-31.

18. Zaigham M, Andersson $\mathrm{O}$. Maternal and perinatal outcomes with $\mathrm{CO}$ VID-19: A systematic review of 108 pregnancies. Acta Obstet Gynecol Scand. 2020;99(7):823-9.
19. Smith V, Seo D, Warty R, Payne O, Salih M, Chin KL, et al. Maternal and neonatal outcomes associated with COVID-19 infection: A systematic review. PLoS One. 2020;15(6):e0234187.

20. Violi F, Pastori D, Cangemi R, Pignatelli P, Loffredo L. Hypercoagulation and antithrombotic treatment in coronavirus 2019: A new challenge. Thromb Haemost. 2020;120(6):949-56.

21. Abbassi-Ghanavati M, Greer LG, Cunningham FG. pregnancy and laboratory studies: a reference table for clinicians. Obstet Gynecol. 2009;114(6):1326-31.

22. Huang C, Wang Y, Li X, Ren L, Zhao J, Hu Y, et al. Clinical features of patients infected with 2019 novel coronavirus in Wuhan, China. Lancet. 2020;395(10223):497-506

23. Tang N, Li D, Wang X, Sun Z. Abnormal coagulation parameters are associated with poor prognosis in patients with novel coronavirus pneumonia. J Thromb Haemost. 2020;18(4):844-7.

24. Thachil J, Tang N, Gando S, Falanga A, Cattaneo M, Levi M, et al. ISTH interim guidance on recognition and management of coagulopathy in COVID-19. J Thromb Haemost. 2020;18(5):1023-6.

25. Kermali M, Khalsa RK, Pillai K, Ismail Z, Harky A.The role of biomarkers in diagnosis of COVID-19 - A systematic review. Life Sci. 2020;254:117788.

26. Thachil J, Longstaff C, Favaloro EJ, Lippi G, Urano T, Kim PY; SSC Subcommittee on Fibrinolysis of the International Society on Thrombosis and Haemostasis. The need for accurate D-dimer reporting in COVID-19: Communication from the ISTH SSC on fibrinolysis. J Thromb Haemost. 2020;18(9):2408-11.

27. Favilli A, Mattei Gentili M, Raspa F, Giardina I, Parazzini F, Vitagliano A, et al. Effectiveness and safety of available treatments for COVID-19 during pregnancy: a critical review. J Matern Fetal Neonatal Med. 2020:114.

28. The American College of Obstetricians / Gynecologist. Society for Maternal-Fetal Medicine (Internet). Último acceso 03/10/2021. Evaluación y tratamiento ambulatorio para mujeres embarazadas con sospecha 0 confirmación de enfermedad por coronavirus 2019 (Coronavirus Disease 2019, COVID-19). 08/25/2020. Disponible en: https://www.acog.org/-/ media/project/acog/acogorg/

29. Kwiatkowski S, Borowski D, Kajdy A, Poon LC, Rokita W, Wielgos M. Why we should not stop giving aspirin to pregnant women during the COVID-19 pandemic. Ultrasound Obstet Gynecol. 2020;55(6):841-3.

30. Gavillet M, Rolnik DL, Hoffman MK, Panchaud A, Baud D. Should we stop aspirin prophylaxis in pregnant women diagnosed with COVID-19? Ultrasound Obstet Gynecol. 2020;55(6):843-4.

31. Magallanes-Garza GI, Valdez-Alatorre C, Dávila-González D, Martínez-Reséndez MF, Sánchez-Salazar SS, Castilleja-Leal F, et al. Rapid improvement of a critically ill obstetric patient with SARS-CoV-2 infection after administration of convalescent plasma. Int J Gynaecol Obstet. 2021;152(3):439-41.

32. Jafari R, Jonaidi-Jafari N, Dehghanpoor F, Saburi A. Convalescent plasma therapy in a pregnant COVID-19 patient with a dramatic clinical and imaging response: A case report. World J Radiol. 2020;12(7):137-41.

33. Valk SJ, Piechotta V, Chai KL, Doree C, Monsef I, Wood EM, et al. Convalescent plasma or hyperimmune immunoglobulin for people with COVID-19: a rapid review. Cochrane Database Syst Rev. 2020;5(5):CD013600.

34. Bates SM, Rajasekhar A, Middeldorp S, McLintock C, Rodger MA, James $\mathrm{AH}$, et al. American Society of Hematology 2018 guidelines for management of venous thromboembolism: venous thromboembolism in the context of pregnancy. Blood Adv. 2018;2(22):3317-59.

35. Thromboembolic disease in pregnancy and the puerperium: Acute management [Internet]. Londres, Reino Unido: Royal College of Obstetricians \& Gynaecologist; abril 2015. Disponible en: https://www.rcog.org. uk/globalassets/documents/guidelines/gtg-37b.pdf

36. Schindewolf $\mathrm{M}$, Gobst $\mathrm{C}$, Kroll $\mathrm{H}$, Recke A, Louwen F, Wolter M, et al High incidence of heparin-induced allergic delayed-type hypersensitivity reactions in pregnancy. J Allergy Clin Immunol. J Allergy Clin Immunol. 2013:132(1):131-9.

37. Manejo de la mujer embarazada y el recién nacido con COVID-19 [Internet]. Madrid, España: Ministerio de Sanidad, Gobierno de España; 17 de junio de 2020: Disponible en: https://www.mscbs.gob.es/profesionales/saludPublica/ccayes/alertasActual/nCov/documentos/Documento_ manejo_embarazo_recien_nacido.pdf

38. Benhamou D, Keita H, Ducloy-Bouthors AS; Obstetric Anaesthesia and Critical Care Club Working Group.Coagulation changes and thromboembolic risk in COVID-19 obstetric patients. Anaesth Crit Care Pain Med. 2020;39(3):351-3.

39. Walker KF, O'Donoghue K, Grace N, Dorling J, Comeau JL, Li W, et al. Maternal transmission of SARS-COV-2 to the neonate, and possible routes for such transmission: A systematic review and critical analysis. BJOG. 2020;127(11):1324-36.

40. Informe semanal de notificación inmediatra de muerte materna. Semana epidemiológica 50 de 2020 [Internet]. México: Secretaría de Salud, Dirección General de Epidemiología. Disponible en: https://www.gob.mx/ cms/uploads/attachment/file/599877/MM_2020 SE50.pdf 